\title{
EGFR-TKI Treatment and Surgical Resection for Oligometastatic NSCLC?
}

\author{
Houke M. Klomp Ingrid Kappers \\ Department of Surgical Oncology, Netherlands Cancer Institute - Antoni van Leeuwenhoek Hospital, Amsterdam, The Netherlands
}

Clinicians who treat patients with lung cancer are familiar with the fact that non-small cell lung cancer (NSCLC) is a heterogeneous disease. Until recently, we could only rely on classic histopathological and radiological staging, and we lacked the ability to use molecular profiles to classify patients into more clinically meaningful subgroups. Novel therapies such as epidermal growth factor receptor tyrosine kinase inhibitors (EGFR-TKIs) for NSCLC have had a transforming effect on both lung cancer research and clinical care.

In this issue of OnKOLOGIE, the article by Levchenko and colleagues focuses on up-front treatment with gefitinib in patients with advanced NSCLC, followed by surgery [1]. The report describes two patients with EGFR mutated tumours showing remarkable responses within 2-3 months of gefitinib treatment. Surgery was performed for minimal residual disease. Although only two cases are described, the treatment approach is interesting and requires further discussion.

Two to four courses of chemotherapy for NSCLC may yield response rates of up to $40-50 \%$, but (near) complete responses are rare [2]. Small-molecule EGFR-TKI, such as gefitinib and erlotinib, are able to induce swift and remarkable responses in selected patients. Gefitinib and erlotinib can be orally administered and block the tyrosine kinase domain of the EGFR, thereby inhibiting downstream signalling pathways involved in cell proliferation, angiogenesis, invasion, metastasis and prevention of apoptosis. (Near) complete responses and dramatic symptomatic relief have been described repeatedly. Responders are presumed to be those with a distinct tumour biology that relies heavily upon signalling via EGFR. They represent a subgroup of around $10-20 \%$ of the European patient population [2-4].

High response rates $(>70 \%)$ can be achieved when selection of patients is applied through molecular EGFR (and KRAS) mutation analysis, which requires pre-treatment tumour tissue [5]. If pre-treatment tissue or EGFR mutation testing is not available, selection of patients for EGFR-TKI treatment can be based on patient-related factors, such as female gender, non-smoking status, Asian ethnicity, and adeno- or bronchioloalveolar carcinoma histology. Such selection is associated with favourable responses in up to $30 \%$ of patients $[5,6]$.

The success of EGFR-TKIs in these subpopulations of NSCLC patients has been bittersweet. Certainly the high response rates and remarkable responses with no identifiable or minimal residual disease are exiting. However, these can hardly be seen as cure. Even dramatic responders eventually relapse, and the mechanism of acquired resistance is unclear. At recent conferences, several molecular strategies to overcome such resistance have been hypothesized which may provide a more sustainable 'cure' [2-7].

The paper of Levchenko et al. [1] describes the aspect of local consolidation therapy to overcome the problem of acquired resistance. To sustain a major response to EGFR-TKIs in patients with 'oligometastatic' NSCLC, surgical resection of the primary tumour (with regional lymph nodes) and a solitary metastasis may therefore be considered.

However, the major problem of this strategy is the identification of patients who may benefit from surgical resection. Selection is highly dependent on the reliability of imaging, not only to evaluate disease extent but also for response assessment. Dissemination in NSCLC is often more widespread than the available imaging techniques show us. Even after complete resection of 'early-stage' NSCLC, long-term survival rates are disappointing. The International Association for the Study of Lung Cancer (IASLC) staging project reported 5year survival of 56 and $38 \%$ for pathologically staged N0 and N1 disease, respectively [8]. Recurrences most often arise at distant sites, confirming the idea that even early-stage operable NSCLC is often a (micro)metastatic disease at diagnosis.

Positron emission tomography (PET) has shown to improve imaging accuracy especially in the identification of

\section{KARGER}

Fax +497614520714

Information@Karger.de

www.karger.com (c) 2009 S. Karger GmbH, Freiburg

Accessible online at:

www.karger.com/onk
Dr. Ingrid Kappers

Netherlands Cancer Institute, Antoni van Leeuwenhoek Hospital

Department of Surgical Oncology

Plesmanlaan 121, 1066 CX Amsterdam, The Netherlands

Tel. +31 20 5122-551, Fax -554

i.kappers@nki.nl 
distant NSCLC spread. As Levchenko et al. appropriately discuss, some studies indicate that surgical resection may be associated with favourable survival in selected patients with stage IV NSCLC. In this group of patients with so-called oligometastatic disease, outcome is highly dependent on selection. Since PET can identify (the extent of) distant metastatic disease more accurately than CT, evaluation by PET/CT will probably improve selection of patients considered for surgical resection.

Clinical observations have been crucial in further research concerning the biological basis of the dramatic responses seen in patients treated with EGFR-TKIs. Despite a better understanding of the molecular heterogeneity of tumours and the complex multidirectional interactions between signalling pathways, the challenge remains not only to establish optimal combinations of novel agents that are effective and well tolerated, but also to integrate novel therapies with other treatment modalities into more effective treatment strategies. Local treatment, such as surgical resection or radiotherapy, may be considered for consolidation therapy after major response to EGFR-TKIs, even in oligometastatic disease. How to select patients for such treatments remains an open question. Careful clinical observations as well as biological studies could help us to devise optimal treatment strategies for this special subgroup of patients.

\section{Conflict of Interest statement}

Dr. Klomp has received an unrestricted research grant from a non-pharmaceutical family fund and from Roche, the Netherlands.

Dr. Kappers has no conflict of interest to disclose.

\section{References}

1 Levchenko EV, Moiseyenko VM, Matsko DE, Iyevleva AG, Ivantsov AO, Yargnian SM, et al.: Down-staging of EGFR mutation-positive advanced lung carcinoma by gefitinib followed by surgical intervention: follow-up of 2 cases. Onkologie 2009:32:674-677.

2 Lynch TJ, Adjei AA, Bunn PA, Jr., Eisen TG, Engelman J, Goss GD, et al.: Summary statement: novel agents in the treatment of lung cancer: advances in epidermal growth factor receptortargeted agents. Clin Cancer Res 2006;12:4365s4371s.
Janne PA: Ongoing first-line studies of epidermal growth factor receptor tyrosine kinase inhibitors in select patient populations. Semin Oncol 2005;32(Suppl 10):S9-15.

4 Kappers I, Klomp HM, Burgers JA, Van Zandwijk N, Haas RL, van Pel R: Neoadjuvant (induction) erlotinib response in stage IIIA non-small-cell lung cancer. J Clin Oncol 2008;26:4205-4207.

5 Shepherd FA, Rosell R: Weighing tumor biology in treatment decisions for patients with non-small cell lung cancer. J Thorac Oncol 2007;2 (Suppl 2): S68-76.
6 Tsao MS, Sakurada A, Cutz JC, Zhu CQ, KamelReid S, Squire J, et al.: Erlotinib in lung cancer - molecular and clinical predictors of outcome. N Engl J Med 2005;353:133-44.

7 Engelman JA, Janne PA: Mechanisms of acquired resistance to epidermal growth factor receptor tyrosine kinase inhibitors in non-small cell lung cancer. Clin Cancer Res 2008;14:2895-2899.

8 Goldstraw P, Crowley J, Chansky K, Giroux DJ, Groome PA, Rami-Porta R, et al.: The IASLC Lung Cancer Staging Project: proposals for the revision of the TNM stage groupings in the forthcoming (seventh) edition of the TNM Classification of malignant tumours. J Thorac Oncol 2007;2:706-714. 